\title{
Simulation of the evolution of concentrated shear layers in a Maxwell fluid with a fast high-resolution finite-difference scheme
}

\author{
Raz Kupferman ${ }^{1, a, *}$, Morton M. Denn ${ }^{\mathrm{b}}$ \\ ${ }^{a}$ Department of Mathematics, Lawrence Berkeley National Laboratory, Mail Stop 50A-2152, Berkeley, CA 94720, USA \\ ${ }^{\mathrm{b}}$ Materials Sciences Division, Lawrence Berkeley National Laboratory and Department of Chemical Engineering, University \\ of California at Berkeley, Berkeley, CA 94720-1462, USA
}

Received 14 July 1998; received in revised form 22 September 1998

\begin{abstract}
We employ a new second-order extension of the Lax-Friedrichs scheme, with incompressibility imposed by means of a projection algorithm, to follow the evolution of the vorticity and stress fields for a Maxwell fluid in a two-dimensional periodic system initialized with two thin and concentrated shear layers. The scheme is simple, efficient, and robust, and is capable, in particular, of resolving large stress gradients. (C) 1999 Elsevier Science B.V. All rights reserved.
\end{abstract}

Keywords: Maxwell; Resolution; Covergence; Shear layers; Stress

\section{Introduction}

Numerical schemes for the solution of viscoelastic flows typically break down when they must resolve large stress gradients. Kupferman and Tadmor [1] recently described a new, computationallyefficient, easily-implementable numerical scheme that, when tested for Newtonian fluids, was found to be immune to spurious instabilities when the flow is under-resolved; i.e., when the gradients become large compared to the discretization [2]. The scheme can be applied to a wide range of differential-type viscoelastic models, and it performed very well when applied to the development of secondary flows of an Oldroyd fluid in a Taylor-Couette geometry [3]. The method employs finite differencing, and is essentially a second-order extension of the classical Lax-Friedrichs scheme [4], with the incompressibility constraint treated by a projection algorithm [5]. It is based on a methodology developed by Tadmor and co-workers [6-9], differing from most modern schemes in that the hyperbolic

\footnotetext{
* Corresponding author.

${ }^{1}$ Present address: Institute of Mathematics, The Hebrew University, Jerusalem, 91904 Israel.
} 
terms are solved by a central scheme that obviates the need to solve a Riemann problem, which for viscoelastic flows in two and three dimensions would be very difficult.

In the present paper we test this new scheme on the classical benchmark problem of a twodimensional doubly-periodic cell with an initial condition that consists of two thin shear layers. The flow for a Newtonian fluid is very unstable and rapidly evolves into a convoluted vortical structure in which large stress gradients are generated. We solve the equations of an upper-convected Maxwell fluid in this geometry for large local Weissenberg numbers; we follow the evolution of the vorticity and the stress, perform a numerical convergence test, assess the order of convergence of the scheme, and obtain an estimate for the error. We test the robustness of the scheme by solving the problem for both large and small Reynolds numbers.

\section{The numerical scheme}

In this section we briefly describe the numerical scheme; for a more detailed presentation see $[1,3]$. We are considering a two-dimensional fluid that satisfies the momentum equations

$$
\frac{\partial \boldsymbol{u}}{\partial t}=-\nabla \cdot(\boldsymbol{u} \boldsymbol{u}+p \boldsymbol{\delta}-\tau)
$$

where $\boldsymbol{u}(\boldsymbol{x}, t)=(u(\boldsymbol{x}, t), v(\boldsymbol{x}, t))$ is the velocity field, $p(\boldsymbol{x}, t)$ is the pressure, $\boldsymbol{\delta}$ is the unit tensor, and $\tau$ is the extra-stress tensor. We consider flow in a doubly periodic unit square, $x \in[0,1) \times[0,1)$; the flow is incompressible, hence

$$
\nabla \cdot \boldsymbol{u}=0 .
$$

The stress $\tau$ is a $2 \times 2$ symmetric tensor assumed to satisfy the upper-convected Maxwell equation

$$
\frac{\partial \tau}{\partial t}=-\nabla \cdot(\boldsymbol{u} \tau)+(\nabla \boldsymbol{u})^{\dagger} \cdot \tau+\tau \cdot(\nabla \boldsymbol{u})-\frac{1}{\lambda} \tau+2 \frac{\eta}{\lambda} \boldsymbol{D}
$$

where $\lambda$ is the relaxation time, $\eta$ the viscosity and $\boldsymbol{D}$ the rate-of-strain tensor. All quantities, including the parameters in the constitutive equation, are assumed to be dimensionless, with lengths measured in units of the box size, velocities in units of a characteristic velocity, time in the ratio of box size to characteristic velocity, and stress in units of the fluid density times the square of the characteristic velocity. In these units the viscosity is the reciprocal of the Reynolds number and $\lambda$ is a global Weissenberg number.

Eqs. (1) and (3) have been written in a conservative form rather than in the more standard convective form, the equivalence between the two being guaranteed by the incompressibility condition Eq. (2). We do so because the numerical scheme is based on a conservative formulation of the equations of motion. Note that only the momentum equation can be written in a fully conservative form. Thus, if we define $\boldsymbol{\psi}(\boldsymbol{x}, t)$ to be the $(2+3)$-dimensional vector whose entries are the two velocity and three independent stress components, its equation of motion has the following general structure:

$$
\frac{\partial \boldsymbol{\psi}}{\partial t}=\frac{\partial \boldsymbol{F}(\boldsymbol{\psi})}{\partial x}+\frac{\partial \boldsymbol{G}(\boldsymbol{\psi})}{\partial y}+\boldsymbol{S}(\boldsymbol{\psi})
$$


where the vectors $\boldsymbol{F}(\psi), \boldsymbol{G}(\psi)$ and $\boldsymbol{S}(\psi)$ are functions of the vector $\psi$ and its derivatives. In the context of conservative systems $\boldsymbol{F}(\psi)$ and $\boldsymbol{G}(\psi)$ are fluxes and $\boldsymbol{S}(\boldsymbol{\psi})$ is a source term.

The unit square is discretized into a Cartesian grid of $N^{2}$ cells of size $\Delta x \times \Delta y$, where $\Delta x=\Delta y=$ $1 / N$. We assume initial data at time $t=t^{\mathrm{n}}$ that represent the values of the velocity components and the three stress components at the centers of the computational cells, which we denote by $\psi_{i, j}^{n}=\left(u_{i, j}^{n}, v_{i, j}^{n},\left(\tau_{x x}\right)_{i, j}^{n},\left(\tau_{x y}\right)_{i, j}^{n},\left(\tau_{y y}\right)_{i, j}^{n}\right.$. The pressure gradient is assumed to be known at the former mid-time; its components are denoted by $\nabla_{x} p_{i, j}^{n-(1 / 2)}$.

The scheme is structurally divided into three steps:

\subsection{Piecewise linear reconstruction}

We first reconstruct a piecewise linear approximation that recovers the point values of the velocity and stress fields inside each cell. In practice, we just need to estimate the first derivatives of the vector $\psi$ at the center of each cell. As shown in [1], it is possible to approximate the derivatives by simple central differences. When sharp gradients are expected it is however advised to use non-linear flux limiters. In the calculations below we used Min-Mod limited derivatives, namely

$$
\begin{aligned}
& \boldsymbol{\psi}_{i, j}^{\prime}=\operatorname{MM}\left(D_{x}^{+} \psi_{i, j}^{n}, D_{x}^{-} \boldsymbol{\psi}_{i, j}^{n}\right) \\
& \boldsymbol{\psi}_{i, j}^{\prime}=\operatorname{MM}\left(D_{y}^{+} \psi_{i, j}^{n}, D_{y}^{-} \psi_{i, j}^{n}\right),
\end{aligned}
$$

where $\psi_{i, j}^{\prime}$ and $\psi_{i, j}^{\prime}$ approximate the $x$ - and $y$-derivatives of $\psi$, respectively, $D_{x}^{ \pm}$and $D_{y}^{ \pm}$are the forward and backward difference operators (e.g., $\left.D_{x}^{+} \psi_{i, j}^{n},=\left(\psi_{i+1, j}^{n}-\psi_{i, j}^{n}\right) / \Delta x\right)$, and MM denotes the Min-Mod limiter

$$
\begin{aligned}
& \operatorname{MM}\left(x_{1}, x_{2}\right)=\min \left(x_{1}, x_{2}\right) \quad \text { if } x_{1}, x_{2}>0 \\
& \operatorname{MM}\left(x_{1}, x_{2}\right)=\max \left(x_{1}, x_{2}\right) \quad \text { if } x_{1}, x_{2}<0 \\
& \operatorname{MM}\left(x_{1}, x_{2}\right)=0 \quad \text { otherwise. }
\end{aligned}
$$

\subsection{Predictor-corrector}

The second step is to evolve the vector $\psi$ to the next time level $t=t^{n+1}$, along with the pressure gradient that has to be evolved to the mid-time $t=t^{n+1 / 2}$. The second-order projection method separates these two tasks by first evolving the vector $\psi$, substituting for the pressure gradient its value at the former mid-time [10]; this gives a provisional velocity field that will generally not satisfy the zerodivergence condition. Projecting this provisional field onto the space of divergence free vector fields returns the velocity field at the next time-level, while the residual of the projection operation is used to update the pressure gradient.

The vector $\psi$ is advanced in time using a two-step predictor-corrector scheme. The predictor step is a first-order Euler scheme that evaluates the fields at the mid-time $t=t^{n+1 / 2}$,

$$
\boldsymbol{\psi}_{i, j}^{n+\frac{1}{2}}=\boldsymbol{\psi}_{i, j}^{n}+\frac{\Delta t}{2}\left[D_{x}^{0} \boldsymbol{F}\left(\boldsymbol{\psi}_{i, j}^{n}\right)+D_{y}^{0} \boldsymbol{G}\left(\boldsymbol{\psi}_{i, j}^{n}\right)+\boldsymbol{S}\left(\boldsymbol{\psi}_{i, j}^{n}\right)\right]
$$


where $D_{x}^{0}$ and $D_{y}^{0}$ denote central difference operators. The predictor step is followed by a corrected step that advances the fields to the time-level $t=t^{n+1}$. A key issue in the scheme is that the updated fields are calculated on a staggered grid, i.e., on a grid that is translated by half a cell with respect to both axes. The staggering is what enables us to account for the hyperbolic terms accurately without having to solve a Riemann problem [11] explicitly. In the context of hyperbolic systems we are averaging over the entire 'Riemann fan', and hence we do not need to track the characteristics. We denote grid functions over the staggered grid by half-integer subscripts; the notation is self-explanatory. The vector $\psi_{i+1 / 2, j+1 / 2}^{n+1}$ at the next time level is given by

$$
\begin{aligned}
\boldsymbol{\psi}_{i+1 / 2, J+1 / 2}^{n+1}= & \mu_{x}^{+} \mu_{y}^{+} \boldsymbol{\psi}_{i, j}^{n}-\frac{\Delta x^{2}}{8} D_{x}^{+} \mu_{y}^{+} \boldsymbol{\psi}_{i, j}-\frac{\Delta y^{2}}{8} D_{y}^{+} \mu_{x}^{+} \boldsymbol{\psi}_{i, j}^{\prime} \\
& +\Delta t\left[D_{x}^{+} \mu_{y}^{+} \boldsymbol{F}\left(\boldsymbol{\psi}_{i, j}^{n+1 / 2}\right)+D_{y}^{+} \mu_{x}^{+} \boldsymbol{G}\left(\boldsymbol{\psi}_{i, j}^{n+1 / 2}\right)+\mu_{x}^{+} \mu_{y}^{+} \boldsymbol{S}\left(\psi_{i, j}^{n+1 / 2}\right)\right],
\end{aligned}
$$

where $\mu^{+}$denotes a forward average in the indicated direction e.g., $\left(\mu_{x}^{+} \boldsymbol{\psi}_{i, j}^{n}=\left(\boldsymbol{\psi}_{i+1, j}^{n}+\boldsymbol{\psi}_{i, j}^{n}\right) / 2\right)$. As explained above, the velocity components of the vector $\psi_{i+1 / 2, j+1 / 2}^{n+1}$ are not the updated velocity components but rather provisional quantities that we denote by $\left(\tilde{u}_{i+1 / 2, j+1 / 2}^{n+1}, \tilde{v}_{i+1 / 2, j+1 / 2}^{n+1}\right)$. The updated velocity is obtained by the last step of our scheme.

\subsection{Projection}

The projection method is based on a theorem that states that any vector field can be uniquely decomposed into the sum of a divergence-free and an irrotational field. This theorem extends to functions defined on a grid, provided that the discrete gradient and divergence operators have been defined appropriately. The error we have made in replacing $\nabla p^{n+1 / 2}$ by $\nabla p^{n-1 / 2}$ is an irrotational vector field, hance the true velocity field can be recovered by subtracting from the provisional field, $(\tilde{u}, \tilde{v})$, its irrotational component. Specifically we need to compute a scalar grid function $\phi_{i j}$ by solving the discrete Poisson equation:

$$
\left(D_{x}^{+} D_{x}^{-} \mu_{y}^{+} \mu_{y}^{-}+D_{y}^{+} D_{y}^{-} \mu_{x}^{+} \mu_{x}^{-}\right) \phi_{i, j}=D_{x}^{-} \mu_{y}^{-} \tilde{u}_{i+\frac{1}{2}, j+\frac{1}{2}}^{n+1}+D_{y}^{-} \mu_{x}^{-} \tilde{v}_{i+\frac{1}{2}, j+\frac{1}{2}}^{n+1} .
$$

The velocity field at the next time level is then given by

$$
\begin{aligned}
& u_{i+1 / 2, j+1 / 2}^{n+1}=\tilde{u}_{i+1 / 2, j+1 / 2}^{n+1}-D_{x}^{+} \mu_{y}^{+} \phi_{i, j}, \\
& v_{i+1 / 2, j+1 / 2}^{n+1}=\tilde{v}_{i+1 / 2, j+1 / 2}^{n+1}-D_{y}^{+} \mu_{x}^{+} \phi_{i, j}
\end{aligned}
$$

whereas the updated value of the pressure gradient is

$$
\begin{aligned}
& \nabla_{x} P_{i+1 / 2, j+1 / 2}^{n+1 / 2}=\mu_{x}^{+} \mu_{y}^{+} \nabla_{x} P_{i, j}^{n-1 / 2}+\frac{1}{\Delta t} D_{x}^{+} \mu_{y}^{+} \phi_{i, j} \\
& \nabla_{y} P_{i+1 / 2, j+1 / 2}^{n+1 / 2}=\mu_{x}^{+} \mu_{y}^{+} \nabla_{y} P_{i, j}^{n-1 / 2}+\frac{1}{\Delta t} D_{y}^{+} \mu_{x}^{+} \phi_{i, j} .
\end{aligned}
$$

We solve the Poisson equation (Eq. (9)) using a fast multigrid algorithm [12]; for periodic systems one could use as well the fast Fourier transform. 
A somewhat inconvenient property of the scheme is that the updated fields at the next time level are given on a different grid from the initial data. One could revert to the original, non-staggered grid at the subsequent time level by repeating the above three steps with backward staggering. A simpler remedy is to interpolate the data back onto the original grid at the end of every time step. (Jiang et al. [13] developed a non-staggered version of a second-order central scheme for systems of hyperbolic conservation laws; it is not clear, however, as to how to apply their approach in combination with the projection algorithm).

The treatment of more complicated boundary conditions, in particular the presence of solid walls, fits naturally into the central scheme methodology; for more detail the reader is referred to [3].

\section{Results}

We implemented the scheme on a Sun Ultra 2 workstation, using a $256 \times 256$ grid. A single timestep takes about $10 \mathrm{~s}$; most of the computation time is consumed by the Poisson solver that solves Eq. (9). The time steps were chosen as large as possible, limited by a bound derived from the CourantFriedrichs-Levy (CFL) condition,

$$
\begin{aligned}
& \max _{i, j}\left[\left|u_{i, j}\right|+\sqrt{2\left|\left(\tau_{x x}\right)_{i, j}+\eta / \lambda\right|}\right] \Delta t<C \Delta x, \\
& \max _{i, j}\left[\left|v_{i, j}\right|+\sqrt{2\left|\left(\tau_{y y}\right)_{i, j}+\eta / \lambda\right|}\right] \Delta t<C \Delta y,
\end{aligned}
$$

where $C=0.48$ is a constant. The CFL condition is a stability constraint that ensures that characteristics will not propagate by more than half a mesh size during a time step.

The initial state consists of two thin and concentrated shear layers, given by the velocity profile:

$$
\begin{aligned}
& u_{0}(x, y)=\tanh \left[\rho\left(y-\frac{1}{4}\right)\right] y \leq \frac{1}{2} \\
& u_{0}(x, y)=\tanh \left[\rho\left(\frac{3}{4}-y\right)\right] y>\frac{1}{2} \\
& v_{0}(x, y)=\delta \sin (2 \pi x)
\end{aligned}
$$

where $\rho$ determines the slope of the shear layer and $\delta$ is the amplitude of the initial perturbation. We assume an initially stress-less medium. The parameter $\mathrm{Wi}=\rho \lambda$ is a local Weissenberg number, which is the product of the dimensional relaxation time and the velocity gradient over the shear layer. The results reported below are for large values of the local Weissenberg number.

In Figs. 1 and 2 we show the evolution of the vorticity $\omega=\partial v / \partial x-\partial u / \partial y$ and the shear stress $\tau_{x y}$ for the parameters $\eta=10^{-4}, \lambda=1 \rho=3$, and $\delta=0.05$; the Reynolds number is $\operatorname{Re}=10^{4}$ and the local Weissenberg number is $\mathrm{Wi}=30$. Each sequence of four images shows the fields at fixed time intervals, $t=0.3,0.6,0.9$, and 1.2 .

Fig. 1 shows initially straight vorticity contour lines that destabilize and evolve into a convoluted pattern of finer and finer structure. The vorticity asymptotically forms a coherent structure of 'macro vortices' of opposite sign [14]. As the solution eventually generates structure on scales smaller than the 

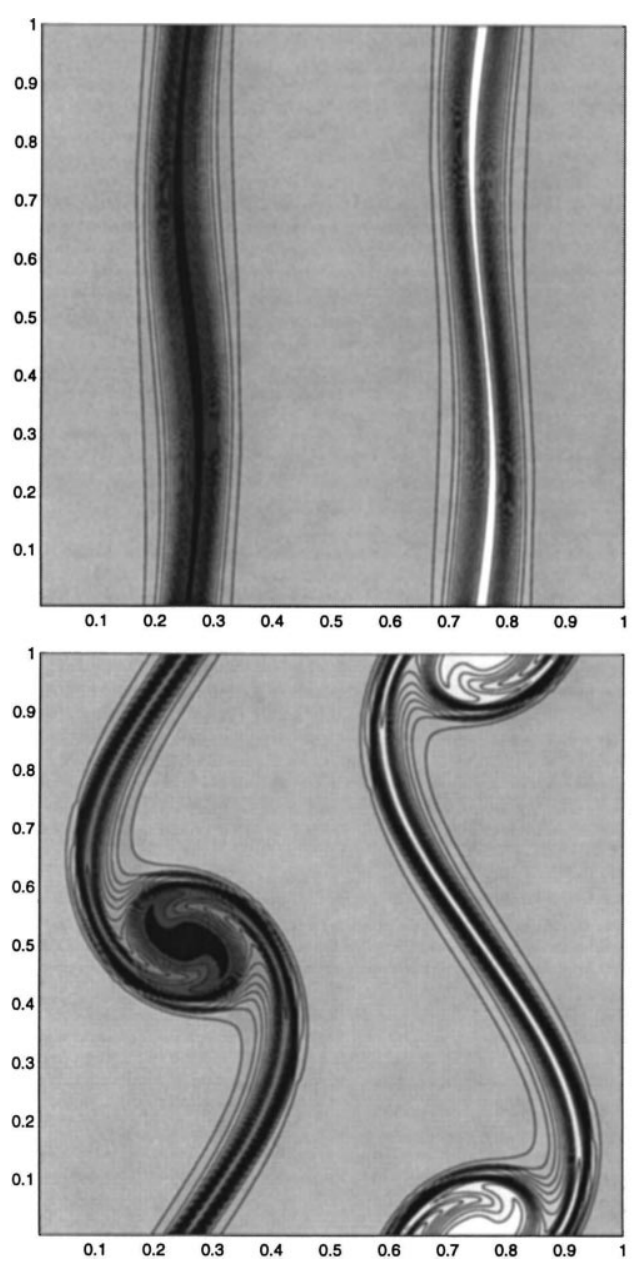
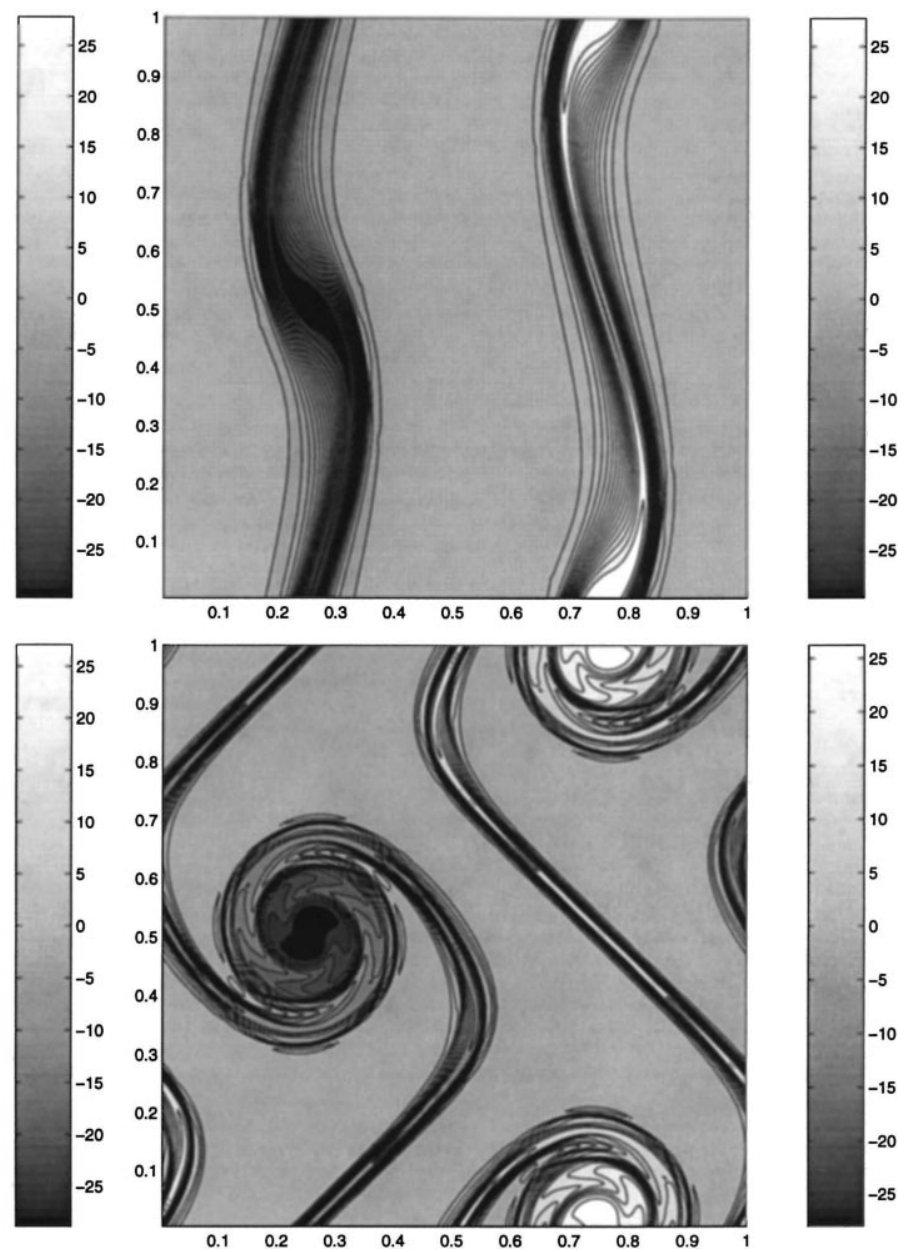

Fig. 1. Contour lines and gray level plots of the vorticity $\omega$ for $\eta=10^{-4}$ and $\lambda=1$ for time (a) $t=0.3$; (b) $t=0.6$; (c) $t=0.9$; (d) $t=1.2$.

mesh size, the computation becomes under-resolved, and accuracy is lost. The loss of accuracy is inevitable; it is a consequence of the physical problem that requires an increasing number of variables to represent an increasingly complex structure. One of the positive properties of this scheme is that the computation does not break down as a result of under-resolution [2]; in fact, the large scale properties may remain quite accurate for some time although, the small scale behavior is no longer correct.

Fig. 2 shows the evolution of the shear stress $\tau_{x y}$; an examination of the normal stresses gives similar results. As expected, stress builds up in the rapidly deforming region; this coincides with the region where the vorticity is large. Since both vorticity and stress are advected by the flow, the high-vorticity and high-stress regions remain roughly coincident as time evolves.

We now analyze the accuracy of our computations. In the absence of an analytical solution to which our results can be compared, we employ a numerical convergence test. To this end the computations were repeated on meshes that are twice and four times coarser (see Figs. 3 and 4). Let $\left(u_{i, j}^{N}, \ldots,\left(\tau_{y y}\right)_{i, j}^{N}\right)$ 

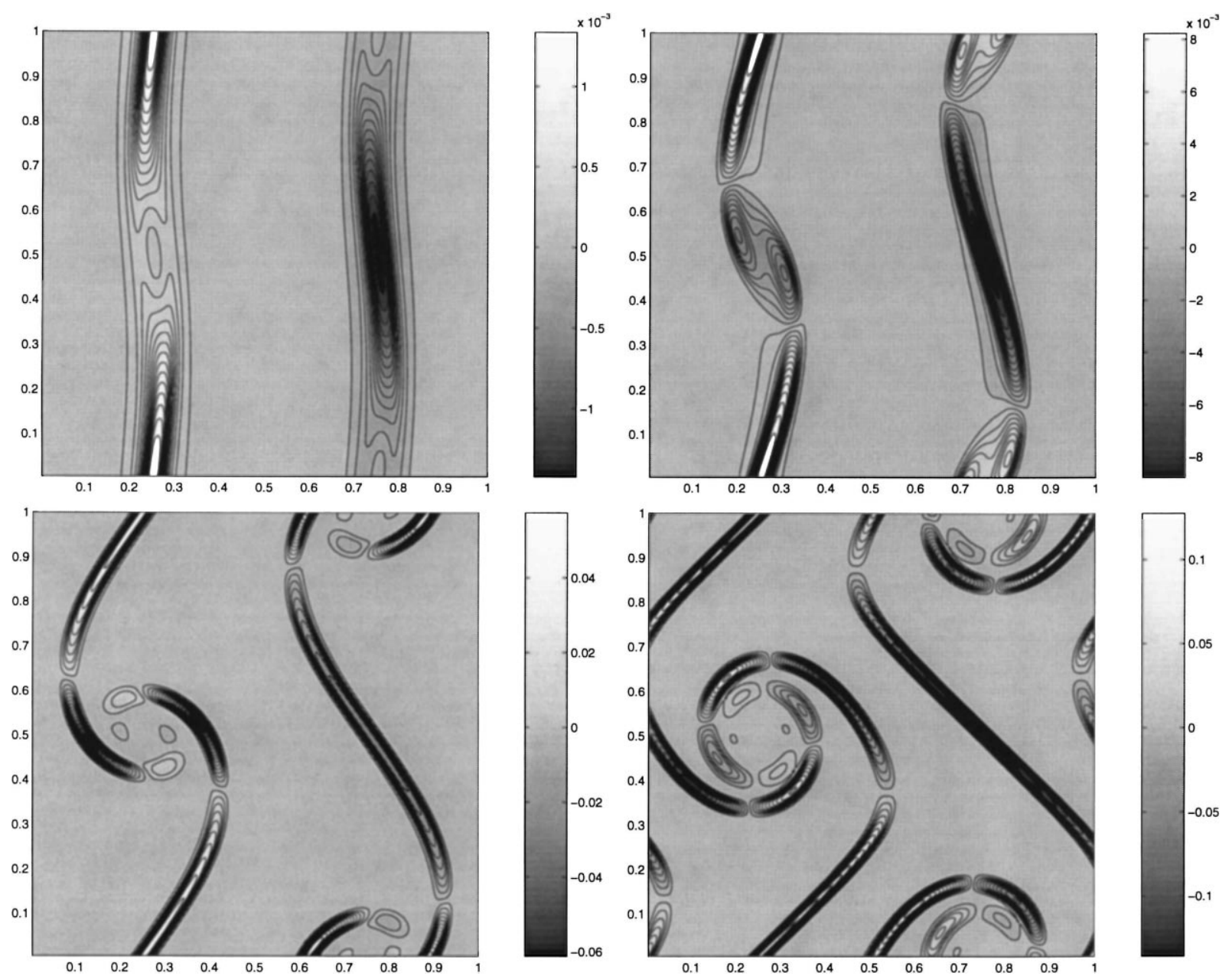

Fig. 2. The stress component $S_{x y}$ for $\eta=10^{-4}$ and $\lambda=1$ for time (a) $t=0.3$; (b) $t=0.6$; (c) $t=0.9$; (d) $t=1.2$.

denote the results of a computation performed on an $N \times N$ grid; then the quantity

$$
e^{N}[u]=\frac{1}{N^{2}} \sum_{i, j=0}^{N-1}\left|u_{i, j}^{N}-u_{i, j}^{2 N}\right|^{2}
$$

for example, can be taken as an estimate of the $L_{2}$-deviation of $u^{\mathrm{N}}$ from the exact solution $u(x, y)$. If a scheme is $\alpha$-order accurate, one expects

$$
\log _{2} \frac{e^{N}[u]}{e^{2 N}[u]} \approx \alpha,
$$

with $\alpha=2$ in the present case. The validity of such error estimates relies on the assumption that the scheme is indeed convergent. 

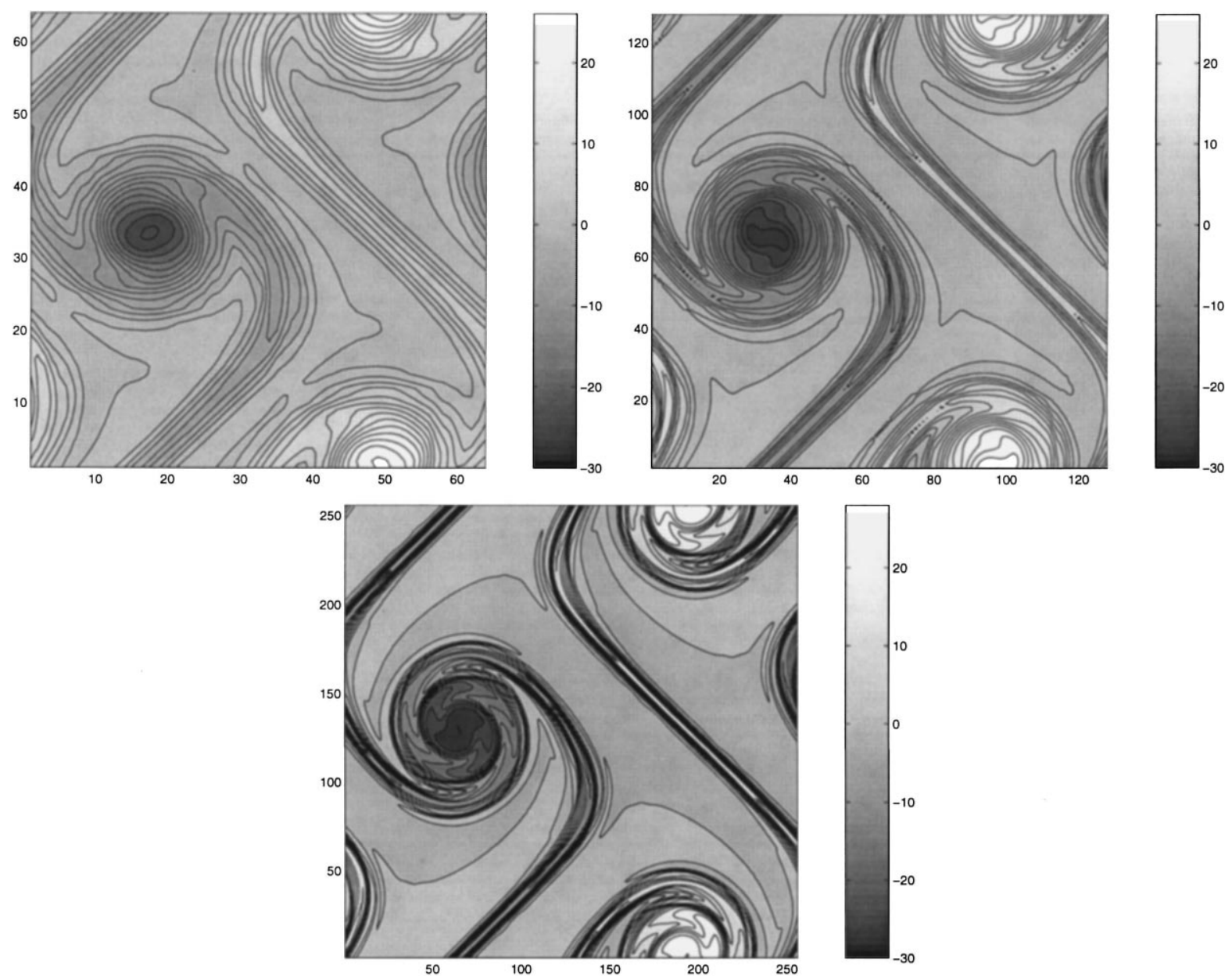

Fig. 3. Convergence test: comparison of the vorticity contour lines at time $t=1.2$ for $\eta=10^{-4}$ and $\lambda=1$, for computations on an $N \times N$ grid with (a) $N=64$; (b) $N=128$; (c) $N=256$.

The results of the analysis are tabulated in Table 1, where we record the error and the convergence rate $\alpha$ for the vorticity $\omega$ and the shear stress $\tau_{x y}$ at various instants of time. The convergence rate is evaluated using Eq. (15). The analysis shows that the estimated convergence rate is slightly below the

Table 1

Convergence test: $L_{2}$-error and convergence rate estimates for the vorticity $\omega$ and the shear $\tau_{x y}$ at five different times

\begin{tabular}{lllllll}
\hline Time & $e^{64}[\omega]$ & $e^{128}[\omega]$ & Rate $(\alpha)$ & $e^{64}\left[\tau_{x y}\right]$ & $e^{128}\left[\tau_{x y}\right]$ & Rate $(\alpha)$ \\
\hline 0.24 & $2.02 \times 10^{-2}$ & $5.99 \times 10^{-3}$ & 1.75 & $3.91 \times 10^{-6}$ & $1.47 \times 10^{-6}$ & 1.41 \\
0.48 & $2.19 \times 10^{-2}$ & $7.09 \times 10^{-3}$ & 1.63 & $1.28 \times 10^{-5}$ & $6.06 \times 10^{-6}$ & 1.08 \\
0.72 & $2.02 \times 10^{-2}$ & $6.66 \times 10^{-3}$ & 1.60 & $2.54 \times 10^{-5}$ & $1.82 \times 10^{-5}$ & 0.48 \\
0.96 & $1.64 \times 10^{-2}$ & $7.55 \times 10^{-3}$ & 1.11 & $6.33 \times 10^{-5}$ & $5.04 \times 10^{-5}$ & 0.33 \\
1.20 & $1.76 \times 10^{-2}$ & $8.19 \times 10^{-3}$ & 1.11 & $9.27 \times 10^{-5}$ & $8.44 \times 10^{-5}$ & 0.13 \\
\hline
\end{tabular}



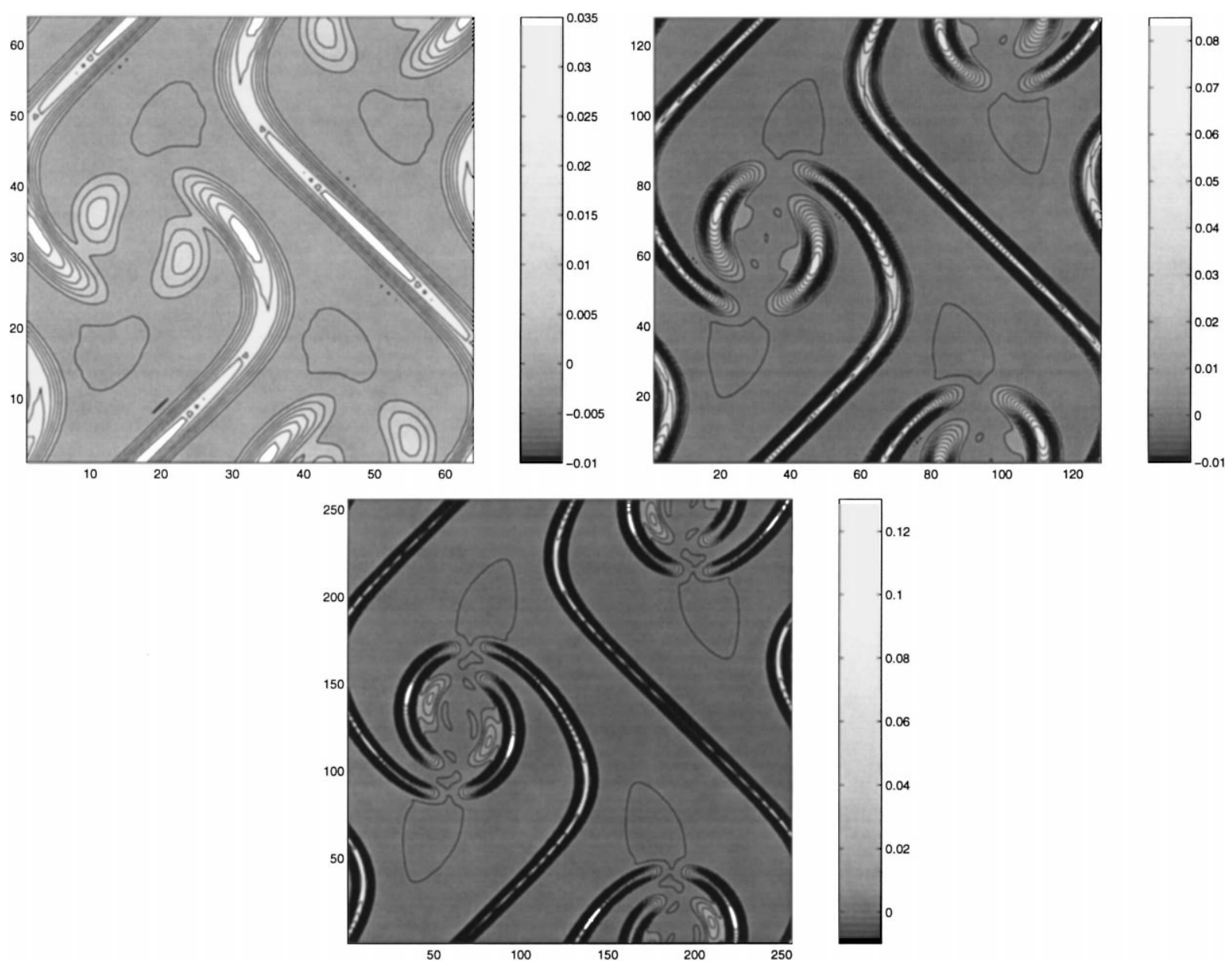

Fig. 4. Comparison of the shear stress contour lines at time $t=1.2$ for $\eta=10^{-4}$ and $\lambda=1$ for computation on an $N \times N$ grid with (a) $N=64$; (b) $N=128$; (c) $N=256$.

expected value of $\alpha=2$ at the very beginning of the simulation, and then gradually decreases with time. The stress convergence is even weaker than the vorticity convergence; at the end of the computation, $t=1.2$, the estimated vorticity convergence rate is nearly first order, while the estimated stress convergence rate is not much greater than zero.

A plausible explanation for the low rate of convergence is that the computational grid is too coarse, so that the converging regime has not been attained. Indeed, the analysis relies on computations performed on a grid as coarse as $64 \times 64$, which is barely sufficient to resolve the initial shear layer, whose gradient is $\rho=30$; it is not surprising then that the accuracy degrades with time as even higher resolution is required. Thus, in order to assess the second-order convergence of our computations, one needs to utilize finer grids: replacing the error estimate $e^{64}[\cdot]$ by an error estimate $e^{256}[\cdot]$, for example. Such computation would be eight times more expensive, though perfectly realizable, even on a workstation. 
Table 2

Same as in Table 1 except that the initial shear layer has a slope of $\rho=10$

\begin{tabular}{lllllll}
\hline Time $(\mathrm{s})$ & $e^{64}[\omega]$ & $e^{128}[\omega]$ & Rate $(\alpha)$ & $e^{64}\left[\tau_{x y}\right]$ & $e^{128}\left[\tau_{x y}\right]$ & Rate $(\alpha)$ \\
\hline 0.24 & $9.89 \times 10^{-4}$ & $2.54 \times 10^{-4}$ & 1.96 & $9.46 \times 10^{-8}$ & $1.74 \times 10^{-8}$ \\
0.48 & $1.34 \times 10^{-3}$ & $2.81 \times 10^{-4}$ & 2.25 & $4.07 \times 10^{-7}$ & $7.05 \times 10^{-8}$ & 2.44 \\
0.72 & $1.56 \times 10^{-3}$ & $3.80 \times 10^{-4}$ & 2.03 & $1.08 \times 10^{-6}$ & $2.52 \times 10^{-7}$ & 2.10 \\
0.96 & $1.82 \times 10^{-3}$ & $4.32 \times 10^{-4}$ & 2.07 & $2.74 \times 10^{-6}$ & $9.98 \times 10^{-7}$ & 1.46 \\
1.20 & $2.11 \times 10^{-3}$ & $7.05 \times 10^{-4}$ & 1.57 & $7.13 \times 10^{-6}$ & $3.62 \times 10^{-6}$ & 0.98 \\
\hline
\end{tabular}

Instead, we repeated the above computations for a different initial condition, this time with an initial shear gradient $\rho=10$. The results of the corresponding convergence analysis are presented in Table 2 . As the flow is easier to resolve, the estimated convergence rate is at least second-order for most of the duration of the simulation. The convergence rate starts decreasing below second-order only around $t=0.9$, due again to the increasing complexity of the flow pattern.

The set of simulations described above corresponds to a flow regime of weak elasticity relative to inertia, and the evolution of the vorticity field is not very different from that governed by inviscid Euler flow. We next demonstrate the wide range of applicability of our scheme by considering a different parameter regime, this time at low Reynolds number. The parameters are $\eta=1$ and $\lambda=2$, while the initial conditions are still given by $\rho=30$ and $\delta=0.05$; that is, $\operatorname{Re}=1$ and $\mathrm{Wi}=60$. The evolution of the vorticity and the shear stress is depicted in Figs. 5 and 6, respectively. In this regime of parameters the fluid behaves essentially as an elastic medium that undergoes a damped oscillatory motion. Consider first the evolution of the vorticity: the vorticity is concentrated initially along the two shear layers (Fig. 5(a)). Each shear layer splits into two shear layers of half intensity that propagate away from each other normal to their main axes. Because of the periodicity these shear layers re-merge, forming almost a reflection symmetric image of the initial state (Fig. 5(d)). That is, at time $t \approx 0.72$ we get back a double shear layer but with reversed velocities. This oscillatory motion is of course damped by the viscosity as evidenced by the decreased intensity of shear layers. The evolution of the shear stress (Fig. 6) parallels the vorticity. The shear layers do not thin because of the essentially-elastic response, since the cycle occurs over a time scale short compared to the relaxation time, although there is considerable damping of the stress because of the large viscosity.

\section{Conclusions}

The evolution of structure in the laminar shear layer for a Maxwell fluid provides a convincing demonstration of the potential applicability of this second-order central difference scheme for the simulation of transient viscoelastic flows with large gradients. Prior applications of this methodology have emphasized high-Reynolds number flows, but the example here demonstrates the robustness with respect to Reynolds number. Furthermore, the methodology is not restricted to a specific set of constitutive relations, and can be easily adapted to other differential constitutive models. The extension to three-dimensional flows is also straightforward. 

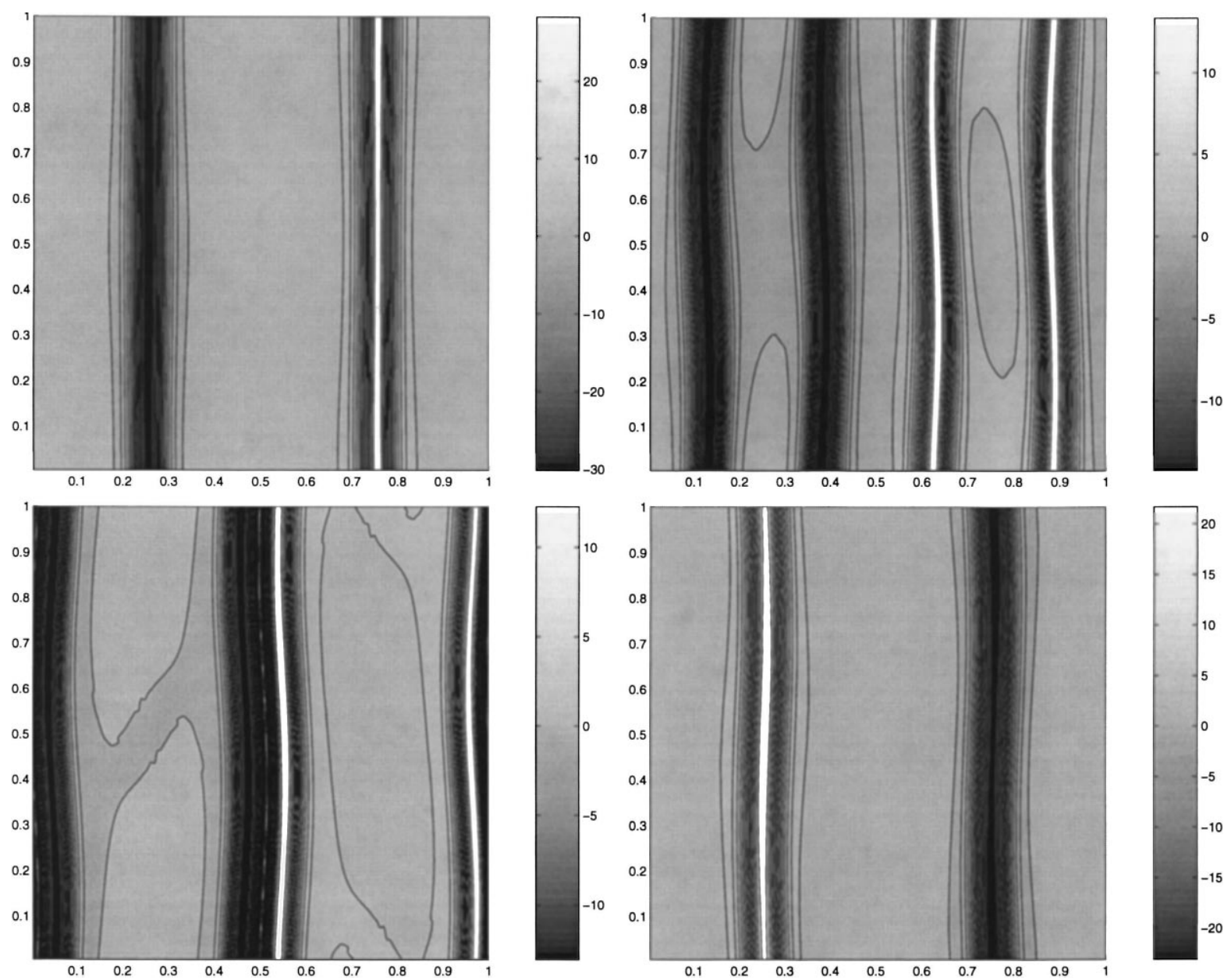

Fig. 5. The vorticity $w$ for $\eta=1$ and $\lambda=2$ for time (a) $t=0$; (b) $t=0.18$; (c) $t=0.3$; (d) $t=0.72$.

For the high Reynolds number case considered here, the flow evolves into a complex structure and cannot be properly resolved at long times without increasing the size of the computational grid; this loss of resolution is manifested by a reduction of the estimated order of accuracy. The remarkable property of the scheme is its robustness with respect to under-resolution; accuracy decreases when the flow is not well resolved, but in a gradual way, rather than causing the computation to break down. It is this feature, combined with the computational efficiency demonstrated in [3], that suggests this scheme for use in more complex flows.

It is of some interest to note that for high Reynolds numbers both the vorticity and the stresses are confined to a relatively small fraction of the plane, and our computation is inefficient in that we expend considerable effort in computing the fields in a region where they are essentially zero, while not sufficiently resolving them in those regions where they are concentrated. One possible remedy would be to develop adaptive mesh refinement algorithms; this has been successfully accomplished in the 

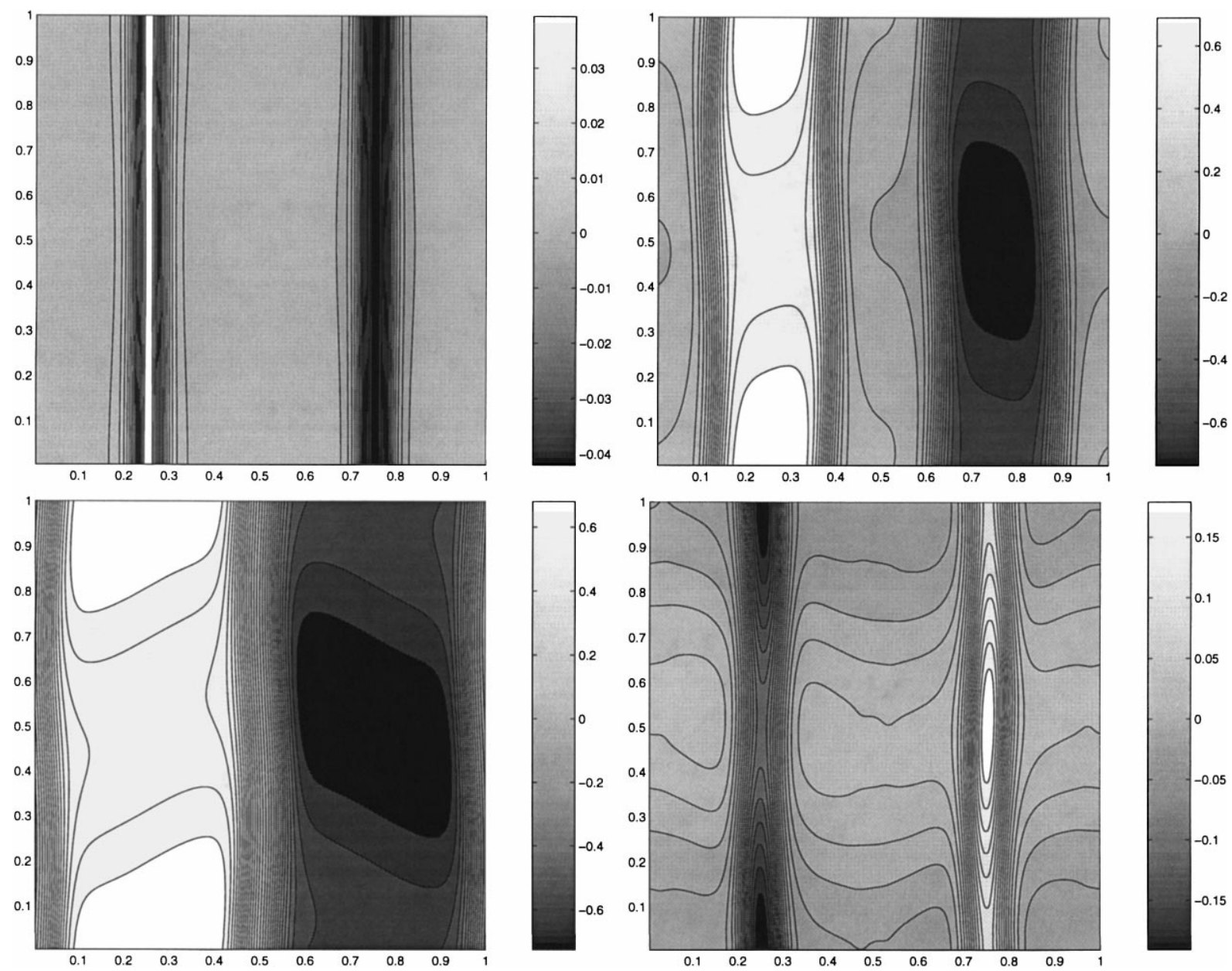

Fig. 6. The stress component $S_{\mathrm{xy}}$ for $\eta=1$, and $\lambda=2$ for time (a) $t=0$; (b) $t=0.18$; (c) $t=0.3$; (d) $t=0.72$.

context of Newtonian fluids for upwind schemes [15], and seems equally applicable within the framework of our central scheme.

\section{Acknowledgements}

We are grateful to Professor Alexandre Chorin for his comments on the manuscript. R.K. was supported in part by the LBNL Directed Research and Development Program, and in part by the Applied Mathematical Sciences Subprogram of the Office of Energy Research, US Department of Energy, and M.M.D. by the Director, Office of Energy Research, Office of Basic Energy Sciences, Materials Science Division, U.S. Department of Energy, both under Contract Number DE-AC03-76SF00098 . 


\section{References}

[1] R. Kupferman, E. Tadmor, A fast high-resolution second-order central scheme for incompressible flows, Proc. Nat. Acad. Sci. USA 94 (1997) 4848-4852.

[2] D.L. Brown, M.L. Minion, Performance of under-resolved two-dimensional incompressible flow simulations, J. Comp. Phys. 122 (1995) 165-183.

[3] R. Kupferman, Simulation of viscoelastic fluids: Couette-Taylor flow, J. Comp. Phys., in press.

[4] P.D. Lax, Weak solution of non-linear hyperbolic equations and their numerical computation, Commun. Pure. Appl. Math. 7 (1954) 159-193.

[5] A.J. Chorin, On the convergence of discrete approximations to the Navier-Stokes equations, Math. Comp. 22 (1969) $745-762$.

[6] H. Nessyahu, E. Tadmor, Non-oscillatory central differencing for hyperbolic conservation laws, J. Comp. Phys. 87 (1990) 408-463.

[7] G.S. Jiang, E. Tadmor, Non-oscillatory central schemes for multidimensional hyperbolic conservation laws, Technical Report 96-36, UCLA, 1996.

[8] D. Levy, E. Tadmor, Non-oscillatory central schemes for the incompressible 2-D Euler equations, Math. Res. Lett. 4 (1997) 1-20.

[9] X.-D. Liu, E. Tadmor, Third-order non-oscillatory central scheme for hyperbolic conservation laws, Numerische Mathematik 79 (1998) 397-425.

[10] J.B. Bell, P. Colella, H.M. Glaz, A second-order projection method for the incompressible Navier-Stokes equations, J. Comp. Phys. 85 (1989) 257-283.

[11] P.D. Lax, Hyperbolic systems of conservation laws and the mathematical theory of shock waves SIAM Publications, Philadelphia, 1972.

[12] W.L. Briggs, A Multigrid Tutorial, Society for Industrial and Applied Mathematics, Philadelphia, PA, 1987.

[13] G.S. Jiang, D. Levy, C.-T. Lin, S. Osher, E. Tadmor. Non-oscillatory central schemes for multidimensional hyperbolic conservation laws, SIAM Journal on Numerical Analysis, 1997,in press,.

[14] A.J. Chorin, Vorticity and turbulence, Springer, New York, 1994.

[15] A.S. Almgren, J.B. Bell, P. Colella, L.H. Howell, M.L. Welcome, A conservative adaptive projection method for the variable density incompressible Navier-Stokes equations, J. Comp. Phys. 141 (1997) 1-146. 